\title{
Ciberperiodismo en el smartphone. Estudio de la multimedialidad, usabilidad, hipertextualidad e interactividad de las aplicaciones de medios nati- vos digitales para smartphone
}

\author{
Carmen Costa SÁNCHEZ \\ Universidade da Coruña (UdC) \\ carmen.costa@udc.es
}

Recibido: $17 / 07 / 2012$

Aceptado: 23/10/2012

\begin{abstract}
Resumen
Los teléfonos móviles ya no son sólo canales para hacer y recibir llamadas. Entre los nuevos usos del medio está el informativo, basado en la web móvil y en las aplicaciones de noticias. La adaptación de las empresas de comunicación a los nuevos soportes y formatos en movilidad no se ha hecho esperar. Medios tradicionales (de todos los sectores, prensa, radio y televisión), así como medios digitales intentan dotar de contenidos a las nuevas pantallas. En la presente investigación se analizan las apps de medios nativos digitales españoles para android e iOS. El objetivo es el de conocer si los medios informativos que nacieron para Internet están optimizando las lecciones aprendidas en su aprovechamiento de la nueva pantalla. Para ello, el análisis se desglosa en cuatro aspectos fundamentales: usabilidad, multimedialidad, hipertextualidad e interactividad. Futuros estudios comparativos en esta línea mostrarán la evolución del empleo del medio móvil con funcionalidad informativa. Este artículo se enmarca en la línea de investigación desarrollada por el Grupo de Cultura y Comunicación Interactiva de la Universidade da Coruña (UdC) acerca de periodismo y dispositivos móviles.
\end{abstract}

Palabras clave: Movilidad, Periodismo, Smartphones, Multimedia, Aplicaciones

Mobile Online Journalism for smartphone. Analysis of multimediality, usability, hypertextuality and interactivity online media apps for smartphone

\begin{abstract}
The cellphones no longer are only channels to do and receive calls. Among the new uses of the channel is the informative use, based in the mobile web and in the news applications. The communication companies adaptation to the new mobile supports and formats is in process. Traditional media (of all sectors, press, radio and television), as well as digital media try to endow of contents to the new screens. In the present research we analyze the Spanish digital media apps for android and iOS. The aim is to know if the media that were born for Internet are optimizing the lessons learnt in their employment of the new screen. This analysis is composed of four fundamental items: usabilility, multimedialidad, hipertextualidad and interactiveness. Future comparative studies will show the evolution of the employment of the mobile platform with informative functionality. This article frames in the main research project developed by the Group of Culture and Interactive Communication of the Universidade da Coruña (UdC) about journalism and mobile devices.
\end{abstract}

Key words: Mobility, Journalism, Smartphones, Multimedia, Applications

Referencia normalizada

COSTA SÁNCHEZ, Carmen (2012): "Ciberperiodismo en el smartphone. Estudio de la multimedialidad, usabilidad, hipertextualidad e interactividad de las aplicaciones de medios nativos digitales para smartphone". Estudios sobre el mensaje periodístico. Vol. 18, núm. especial octubre, págs.: 243-251. Madrid, Servicio de Publicaciones de la Universidad Complutense.

Sumario: 1. Introducción. Un nuevo canal para el periodismo. 2. Metodología 3. Análisis de los resultados. 4. Conclusiones 5. Referencias bibliográficas. 


\section{Introducción. Un nuevo canal para el periodismo}

La combinación de movilidad e Internet ha surtido éxito. Estamos inmersos en un nuevo contexto comunicativo que bien pudiéramos bautizar como la era de los smartphones.

En nuestro país, el empleo del smartphone prácticamente iguala al del teléfono móvil tradicional (un 46,3\% de tasa de penetración, frente al 53,7\%) y la banda ancha móvil encabeza el crecimiento de la demanda de banda ancha en España en 2011, a pesar de la crisis (según recoge el informe La sociedad de la información en España 2011 de la Fundación Telefónica).

La implantación y penetración social de una nueva tecnología comunicativa que resulta ampliamente aceptada altera y transforma los hábitos comunicativos de las personas. Ligado a ello, se producen cambios en los usos informativos que los ciudadanos pueden realizar del nuevo medio.

En el caso de la tecnología móvil, las modificaciones en nuestros hábitos comunicativos son ya palpables: el teléfono nos sirve para actividades diversas, más allá de para hacer o recibir llamadas o SMS. Las finalidades comunicativas convergen en el nuevo dispositivo: comunicarse, informarse, trabajar o atender cuestiones laborales, entretenerse, divertirse, formarse, etc.

Respecto a las dinámicas informativas, los dispositivos móviles (tabletas y smartphones) se están consolidando como herramienta para informarse. En datos del estudio How mobile devices are changing community information environments del Pew Research Center, se recoge que el $47 \%$ de los americanos adultos usan sus teléfonos y tabletas para obtener noticias locales e información. En datos del IV Estudio Anual IAB Spain Mobile Marketing (de Iab Spain Research y The Cocktail Analysis), leer la prensa es la cuarta actividad realizada a diario en la Internet móvil después de chatear, usar el correo electrónico y acceder a redes sociales y de un total de 13 posibilidades.

La adaptación de las empresas de comunicación a los nuevos soportes y formatos en movilidad no se ha hecho esperar. Medios tradicionales (de todos los sectores, prensa, radio y televisión), así como medios digitales intentan dotar de contenidos a las nuevas pantallas.

Desde el punto de vista empresarial, la oferta de una aplicación para móviles, además de un nuevo servicio a los ciudadanos, puede suponer una interesante vía de consolidación de la marca y un nuevo canal para obtener ingresos publicitarios. La fidelidad que aporta la app en comparación con la web supone un valor añadido: si el usuario dispone de la aplicación, accederá al medio desde dicha plataforma que ya tiene permanentemente en pantalla, evitando la posibilidad de entrar/no entrar en la web, o, al menos, por medio de ambas.

"Todos los grupos con los que nos entrevistamos tienen ya un departamento, dentro del organigrama general, dedicado al mantenimiento de los contenidos móviles e integrados por una plantilla (de entre una y dos personas) dedicada, exclusivamente, al dispositivo móvil" (Sánchez Valverde et al., 2010: 3).

Al ritmo de difusión que está consiguiendo la nueva tecnología, el mercado de usuarios que genera resulta muy atractivo para las empresas informativas y las de anunciantes. 
"El ritmo al que crecen los usuarios de estos servicios, el abanico de posibilidades que se están abriendo desde el punto de vista tecnológico, y el significativo nivel de ingresos que están generando, de forma generalizada, los contenidos móviles han hecho sospechar que, lo que hoy es sólo una salida más, puede llegar a convertirse, en un tiempo relativamente breve, en uno de sus motores más importantes, junto con las ediciones web" (Sánchez Valverde et al., 2010: 15).

Los medios nativos digitales constituyen parte interesada en el fenómeno en la medida en que consoliden un nuevo canal con el que acercarse a los ciudadanos. Salaverría (2008) subraya que se trata de un mercado inmaduro y frágil, caracterizado por las dificultades de conseguir financiación de los anunciantes y a menudo ignorado por las instituciones a la hora de elaborar la agenda mediática.

Dos son los desafíos que enfrentan dichas publicaciones: en primer lugar, el mercado de las noticias online sigue estando dominado por los grandes grupos mediáticos y, en segundo lugar, el mercado de la publicidad online está dominado por unos pocos pero destacados actores como Google, lo que dificulta la capacidad de las empresas de pequeño y mediano tamaño a la hora de generar importantes beneficios (Bruno y Nielsen, 2012). Por todo ello, tan sólo la supervivencia ya es un éxito.

En el panorama mediático español, encontramos sin embargo, algunas excepciones a la norma vigente. El portal catalán Vilaweb (http://www.vilaweb.cat/) lleva más de quince años en funcionamiento, siendo de los primeros medios nativos digitales que aparecen en España.

En efecto, el desconocimiento de la marca de estos nuevos media supone un obstáculo añadido para unas publicaciones que nacen con pocos recursos y sin un nombre de referencia. Por ello, resultan de interés dos cuestiones. Primeramente, ¿contemplan el dispositivo smartphone como un canal de interés para su estrategia de negocio? En segundo lugar, siendo empresas que han nacido para la web, ¿cómo se están adaptando al medio móvil?

\section{Metodología}

La presente investigación realiza una aproximación a las aplicaciones para iOS y Android de los medios nativos digitales españoles. El objetivo final es el de conocer cuál es el modelo que están aplicando las empresas periodísticas online para informar y relacionarse con sus usuarios a través del smartphone.

La muestra de estudio estuvo conformada por un total de diez aplicaciones de medios nativos digitales, cinco para iOS y cinco para android.

Ante la dificultad de no disponer de un directorio de medios nativos digitales, acotamos la muestra de estudio a las aplicaciones periodísticas para móviles de medios nativos digitales de los controlados por OJD interactiva y de aquellos que se han hecho un hueco en el panorama informativo español. En total, se analizaron diez aplicaciones, correspondientes a: Lainformacion, Elconfidencial, Vilaweb, Ara.cat y Publico. Nuevas iniciativas nacidas recientemente, como Eldiario.es, carecen de aplicaciones para el dispositivo.

Cabe matizar que Publico es un medio que no es puramente nativo digital si bien a causa de la crisis económica, se convierte exclusivamente en medio online. Por su parte, Ara.cat saca prácticamente en simultáneo la versión en papel y digital. Partiendo 
de análisis previos centrados en aplicaciones periodísticas o informativas en dispositivos móviles (Canavilhas, 2009; Navarro y Lisboa, 2012), se elaboró una ficha de análisis que revisaba los siguientes apartados:

Diseño de portada. Estructura de la página principal de la aplicación desde la que se accederá a los contenidos de interés.

Recursos multimedia. Presencia de fotografías en las noticias, galerías fotográficas o de videos, radios o podcasts.

Usabilidad. En la navegación por la aplicación, analizada conforme a: presencia de menú de secciones, navegación dentro de la misma sección, claridad en la estructura de navegación, etc.

Participación de los usuarios. En redes sociales, comentando o votando noticias o un paso más allá, produciendo contenidos en su nueva faceta de prosumers.

Personalización de los contenidos. Opciones de personalizar contenidos ofrecidas por la aplicación.

Hipertextualidad. Característica derivada del periodismo digital basada en la utilización de enlaces o hipervínculos para relacionar los contenidos y permitir acceder a un mensaje más rico y complejo.

Otros elementos de interés. Buscador interno, formatos publicitarios predominantes, modelos de negocio, etc.

\section{Análisis de los resultados}

Los resultados serán, en primer término descriptivos, según los elementos de análisis que se han mencionado previamente. Como el análisis cuantitativo no ha lugar, siendo tan reducido el universo-muestra de estudio, la reflexión cualitativa nos permitirá adentrarnos en las particularidades de cada caso.

Así, en cuanto al diseño de portada, cabe señalar que las aplicaciones de los medios analizados estructuran la pantalla inicial en tres partes:

Una parte superior, donde se ubica la cabecera, con el nombre del medio y los colores y tipografías corporativas. Es donde se sitúa el buscador interno si es que el medio dispone de él.

Una parte central, donde se ubican los titulares de portada. Suelen estar acompañados de una pequeña fotografía que ilustra la noticia.

Una parte inferior, donde suelen ubicarse el menú de secciones, así como las herramientas que facilitan la interacción/ participación del usuario.

La portada se configura en la mayor parte de los casos como una sucesión de titulares ordenados por fecha y hora de actualización de la información. Se acompañan a la izquierda de una pequeña fotografía que luego forma parte de la noticia seleccionada. La "maquetación" es, por tanto a dos columnas. El criterio de jerarquía de los titulares es la actualización de la información, es decir, se prima en la configuración de la portada el valor-noticia de la actualidad.

De las analizadas, la portada más estructurada es la del medio catalán Ara.cat, que, además de los titulares destacados, incluye galerías fotográficas y sección de meteorología en la portada, para a continuación, aportar los principales titulares agrupados por secciones. 
La portada de Público para android supone la única excepción a la norma antes mencionada, pues la estructura de tres espacios presenta una variación: el menú de secciones se ubica bajo la cabecera en lugar de en el tercer espacio de los tres comentados.

Lo habitual también es que en portada no todas las secciones sean visibles, sino que haya que pulsar en algún icono para ver el menú completo.

En Ara.cat y en Elconfidencial aparecen en portada titulares que carecen de sentido completo y que terminan en puntos suspensivos, con lo que es necesario pulsar en la noticia para conocer su contenido completo.

En cuanto a la presencia de recursos multimedia, sólo las aplicaciones de Lainformacion para android y para iOS así como las de Ara.cat para ambos sistemas presentan galería de fotos y de videos. No hay podcast ni contenidos de audio como recursos para completar las informaciones.

En relación con la usabilidad, vemos los resultados en función de los ítems de análisis planteados:

- La opción de ampliar el tamaño del texto la encontramos en tres de los medios analizados tanto para iPhone como para android. Estos son: Ara.cat, Vilaweb y Elconfidencial.

- La opción de ampliar el tamaño de las fotografías, la encontramos en un solo medio de los analizados, Ara.cat, versiones ios y android.

- La navegación dentro de la misma sección, sin volver al menú principal, para cambiar de noticia sí es una opción extendida pues está presente en cuatro de los medios analizados para ambas plataformas, todos a excepción de Elconfidencial.

- El cambio de sección, una vez, estás dentro de una noticia en concreto, es posible sólo en uno de los medios analizados, en concreto Público, que mantiene el menú de secciones dentro de cada noticia.

En referencia a la interacción de los usuarios que permiten las aplicaciones, por lo general, enviar el contenido de la noticia por correo electrónico (texto e imagen) es la opción que más está presente en las aplicaciones analizadas, seguida de la posibilidad de compartir contenidos en las redes sociales Facebook y Twitter.

La posibilidad de comentar noticias es minoritaria, al igual que la opción de votar noticias o de generar contenidos propios que, como parte de la esencia del periodismo ciudadano, estaría llamada a ser una alternativa de obligada referencia.

En resumen, las posibilidades de participación-interacción son las siguientes:

\begin{tabular}{|l|c|c|c|c|c|c|}
\hline & Votar & Comentar & Fcb & Twitter & E-mail & Contenidos \\
\hline iOS & & & & & & \\
\hline Ara.cat & & $\mathrm{X}$ & $\mathrm{X}$ & $\mathrm{X}$ & $\mathrm{X}$ & \\
\hline Vilaweb & & & & & & \\
\hline El confidencial & & $\mathrm{X}$ & & $\mathrm{X}$ & $\mathrm{X}$ & \\
\hline Publico & & & $\mathrm{X}$ & $\mathrm{X}$ & $\mathrm{X}$ & \\
\hline La informacion & & & $\mathrm{X}$ & & $\mathrm{X}$ & \\
\hline Android & & & & & & \\
\hline Ara.cat & & $\mathrm{X}$ & $\mathrm{X}$ & $\mathrm{X}$ & $\mathrm{X}$ & \\
\hline Vilaweb & & & $\mathrm{X}$ & $\mathrm{X}$ & $\mathrm{X}$ & \\
\hline
\end{tabular}




\begin{tabular}{|l|l|l|l|l|l|l|}
\hline El confidencial & & $\mathrm{X}$ & $\mathrm{X}$ & $\mathrm{X}$ & $\mathrm{X}$ & \\
\hline Público & & & $\mathrm{X}$ & $\mathrm{X}$ & $\mathrm{X}$ & \\
\hline La información & & & $\mathrm{X}$ & & $\mathrm{X}$ & \\
\hline
\end{tabular}

Tabla 1. Posibilidades de interacción del usuario en cada una de las aplicaciones analizadas. Elaboración propia.

Otras opciones de interactividad además de las planteadas pudieron encontrarse en dos casos, en concreto, en las aplicaciones de Ara.cat y de Público para android, donde pueden compartirse contenidos en Google + o también a través de las redes sociales para móviles Whatsapp o ChatON, no así en sus equivalentes para iPhone.

En cuanto a los rankings de contenidos, es decir, jerarquización de contenidos según las preferencias de los usuarios encontramos dos grandes tipos: lo más visto en Ara.cat- y las más comentadas (en Elconfidencial y Vilaweb).

Yendo un paso más allá y buscando opciones de personalización, la única herramienta que permite personalizar en cierta medida las aplicaciones es la posibilidad de crear una sección de Favoritos, con los contenidos que el usuario haya escogido previamente. Esto es permitido en todas las aplicaciones para android de los medios analizados, también en las de iOS. Posibilidades de personalizar menús o diseño de secciones, así como la presentación de las informaciones no se ofrecen en las aplicaciones estudiadas.

Con respecto a la hipertextualidad, el análisis se hacía basándose en la presencia de enlaces a contenidos de la web del medio o a contenidos externos. Ambos elementos se trabajan en todas las aplicaciones analizadas a excepción de Elconfidencial para android, donde no se han encontrado hipervínculos que conecten nodos de información (cuando sí los hay en idénticas informaciones para iOS).

En referencia a contenidos premium, estos sólo están a disposición del usuario en el medio Ara.cat. Vilaweb dispone de otra aplicación, denominada MesVilaweb para usuarios registrados que colaboran económicamente con la supervivencia del medio. Elconfidencial tiene dos cuadernillos especializados, pero son de acceso gratuito: Cotizalia, en clave económica y Vanitatis, en clave rosa y sociedad.

Por lo que respecta a otros elementos de análisis, sólo presentan buscador interno las aplicaciones de Vilaweb y Lainformacion. Con respecto a los formatos publicitarios, se ha encontrado publicidad en dos de los medios analizados. Los formatos predominantes son el banner (en los menús superior e inferior) e interstitials.

\section{Conclusiones}

El teléfono móvil ya no es sólo una herramienta para hacer y recibir llamadas o enviar mensajes de texto. Los estudios cuantitativos sobre uso de teléfonos inteligentes así lo revelan:

"Two-thirds of cellphone users take advantage of mobile phone features such as texting, e-mailing, web browsing and "apps" (software applications that allow mobile device users to play games, access web content and access media or data). Only a third of Americans now say they use their cellphones just for phone calls, according to the survey" (Purcell et al., 2011: 2). 
Las aplicaciones de medios informativos pasan a incorporarse a las estrategias de negocio de los medios tradicionales y de los cibermedios. Ante el nuevo panorama, se requieren estudios que muestren cómo se están utilizando las nuevas posibilidades informativas que aportan las apps por parte de las empresas periodísticas.

Del análisis realizado, podemos concluir que:

En primer lugar, el criterio que está organizando la jerarquía de los temas en la portada principal y en la de las secciones es la actualidad, de modo que las que fueron primeras noticias van siendo desplazadas a últimos lugares de portada a medida que van llegando otras más recientes. La instantaneidad se confirma como elemento predominante del proceso de gatekeeping.

Por otra parte, la gran cantidad de temas en portada (superan los 30 en la mayoría de los casos), así como la ausencia del criterio temático (o por secciones) como criterio clasificador de los mismos convierte a las aplicaciones en un medio rápido para informarse de la última hora, más que para buscar información sobre un asunto en concreto.

Así, el soporte móvil está siendo concebido como un medio adecuado para estar informado de "lo último", para echar un vistazo a los titulares del día. El tamaño de pantalla tampoco es favorable a una lectura de larga duración y continuada que sería necesaria para una tarea informativa en profundidad.

Por otra parte, la subexplotación de los recursos multimedia tampoco contribuye en este sentido. No se recurre a galerías de fotografía ni a recursos de audio, video o incluso, infografía. Dichas herramientas -que, como decimos, apenas están siendo empleadas- podrían facilitar el esfuerzo informativo del ciudadano, completando las noticias con otros recursos no textuales, que sí pueden ser muy adaptables al medio móvil.

Además, otro aspecto que convendría revisar son las insuficientes herramientas de interactividad, participación o interacción de los usuarios

En cuanto a la interactividad y opciones de personalización, la opción de envío de la noticia por correo electrónico es, junto a la opción de compartir contenidos por medio de las redes sociales Facebook o Twitter, las posibilidades predominantes. En este sentido, se encontraron diferencias favorables a las aplicaciones para android (más canales para compartir contenidos).

Las opciones de personalización de todas las aplicaciones analizadas son inexistentes, de donde se puede concluir que el medio móvil está descuidando las potencialidades de personalización que constituyen uno de los principales puntos fuertes del dispositivo, que es, por encima de todo, un medio de uso personal.

Si estableciéramos una gradación en la escala de las posibilidades de interacción del usuario que fuese de menos a más, encontraríamos que nos hallamos en las etapas más elementales de una evolución que es probable veamos avanzar con el paso del tiempo.

Las opciones para fomentar el rol del prosumer (Toffler, 1980), que en este caso confluiría con las finalidades del periodismo participativo o periodismo ciudadano no son, por el momento al menos, buscadas desde las aplicaciones analizadas.

En ambos sentidos, se está yendo por delante preconizando las bondades y potencialidades del fenómeno en la teoría respecto a la práctica, algo ya avanzado por otros autores (Canavilhas, 2009). 
En referencia a la usabilidad, hay aspectos que están funcionando bien (navegación intrasecciones, por ejemplo) pero hay otros que cabría mejorar: la navegación entre secciones sin pasar por el menú principal, así como la opción de ampliar el tamaño de las fotografías y del texto, o la presencia de un buscador interno. La hipertextualidad, heredada del medio web, también está funcionando correctamente.

Estamos, según creemos, ante una primera etapa en la evolución del nuevo medio a nivel informativo. Coincidimos con Canavilhas y Cavallari (2011: 65) cuando afirman que "a pesar desta melhoria geral, registrada entre 2009 e 2011, as plataformas móveis atuais possuem características técnicas ainda pouco exploradas, que permitem ofrecer tipos realmente novos de produtos informativos".

Las aplicaciones imitan los aciertos del ciberperiodismo, pero todavía no han explorado ni aprovechado las potencialidades propias de la participación, la personalización o la multimedialidad que podrían ofrecer a sus usuarios. Próximos estudios a nivel comparativo podrán evidenciar si se está avanzando en este modelo informativo anytime everywhere.

\section{Referencias bibliográficas}

BRUNO, Nicola y NIELSEN, Rasmus Kleis (2012): Survival is Success: Journalistic Online Start-Ups in Western Europe. Oxford, Reuters Institue for the Study of Jornalism. Disponible en http://reutersinstitute.politics.ox.ac.uk/

CANAVILHAS, Joao (2009): “Contenidos informativos para móviles: estudio de aplicaciones para iPhone". Revista Textual \& Visual Media, 2, pp. 61-80. Madrid.

CANAVILHAS, Joao y CAVALLARI, Douglas (2011): "Jornalismo para plataformas móveis de 2008 a 2011: da autonomia à emancipação". Líbero, vol. 14, nº 28, pp. 53-66. São Paulo.

NAVARRO, Héctor y LISBOA NEVES, Alysson (2012): "mPeriodismo. Introducción a las Apps de noticias para dispositivos móviles". Congreso Internacional AE-IC: Comunicación y Desarrollo en la Era Digital. Tarragona, 18-20 enero de 2012. Disponible en: http://www.aeic2012tarragona.org/comunicacions _cd/ok/104.pdf. [fecha de consulta: 10 de septiembre de 2012].

PURCELL, Kristen; RAINIE, Lee; ROSENSTIEL, Tom y MITCHELL, Amy (2011): Spring 2011 iPad survey results. Reynolds Journalism Institute -DPA. Disponible en http://rjionline.org/news/rji-dpa-spring-2011-ipad-survey-results[fecha de consulta: 08 de septiembre de 2012]

SALAVERRÍA, Ramón (2008): "La investigación sobre ciberperiodismo en España: tendencias, resultados y perspectivas". En LÓPEZ, Guillermo (ed.): Comunicación local y nuevos formatos periodísticos en Internet: cibermedios, confidenciales y weblogs. Valencia, Servei de Publicacions de la Universitat de València. pp. 15-34. Disponible en http://www.cibermediosvalencianos.es/comloc/Salaverria.pdf [fecha de consulta: 10 de septiembre de 2012].

SÁNCHEZ VALVERDE, Ester y AGUADO, Juan Miguel (2010): "Dispositivos móviles y convergencia digital en los grupos de comunicación españoles: la perspec- 
tiva de los profesionales". II Congreso Internacional AE-IC Comunicación y Desarrollo en la Era Digital. Málaga, 3-5 febrero de 2010

TOFFLER, Alvin (1980): Future Shock. New York, William Morrow and Co.

\section{Carmen COSTA SÁNCHEZ}

carmen.costa@udc.es

Universidade de Santiago

Facultade de Ciencias da Comunicación

Campus de Elviña

15071 A Coruña

Carmen Costa Sánchez es Doctora en Comunicación por la Universidade de Santiago de Compostela. Desarrolla su labor docente e investigadora en la Universidade da Coruña, donde imparte diversas materias. Entre sus líneas de investigación están la Comunicación Móvil, la Comunicación Corporativa y las nuevas estrategias de comunicación multiplataforma. 